\title{
Levantamento de Doses em Tomografia Computadorizada em Protocolos de Crânio e Tórax
} Survey of Computed Tomography Doses in Head and Chest Protocols

\author{
Giordana Salvi de Souza ${ }^{1}$, Ana Paula Pastre Froner ${ }^{1}$, Ana Maria Marques da Silva1 \\ ${ }^{1}$ Núcleo de Pesquisa em Imagens Médicas, Faculdade de Física, PUCRS, Porto Alegre, Brasil
}

\begin{abstract}
Resumo
Pesquisas sobre estimativas de dose para diferentes modalidades de imagens médicas mostram variações significativas para o mesmo exame em distintas instituições de saúde ou para grupos de pacientes com características semelhantes. Para assegurar que as doses estão otimizadas, níveis de referência diagnóstica (NRD) devem ser determinados, possibilitando a identificação de procedimentos fora dos padrões ideais. O objetivo deste estudo é apresentar um levantamento retrospectivo de indicadores de dose em exames de tomografia computadorizada de crânio e tórax, em termos de DLP (Dose-Length Product) e dose efetiva, para pacientes adultos e pediátricos, comparando-os com NRD encontrados na literatura. $O$ estudo foi realizado com dados de 293 pacientes submetidos à tomografia computadorizada de crânio e 146 de tórax, divididos em faixas etárias $(0-1 ; 1\} 5 ; 5\} 10 ; 10-15 ;>15)$. Para crânio, o terceiro quartil do DLP e doses efetivas são, em sua maioria, maiores do que os NRD da literatura, principalmente para crianças de $5 \dashv 10$ anos (800 mGy.cm; 3,3 mSv) e 10- 15 anos (969 mGy.cm; 2,6 mSv). Para exames de tórax, apresar dos DLP serem próximos dos NRD, uma alta variabilidade é observada, e as doses efetivas são maiores para todas as idades. Conclui-se que o levantamento de indicadores de dose em exames de tomografia computadorizada e sua comparação com NRD constituem uma importante ferramenta para a otimização de doses em pacientes.
\end{abstract}

Palavras-chave: dosimetria; tomografia computadorizada; dose efetiva; nível de referência diagnóstica.

\begin{abstract}
Surveys of dose estimates from different medical imaging modalities highlight significant variations between healthcare facilities for the same examination and similar patient groups. To ensure that doses are optimized, diagnostic reference levels (DRL) must be determined, allowing the identification of procedures that are out of optimal standards. The aim of this study is to present a retrospective survey of dose indicators in head and chest CT scans, in terms of Dose-Length Product (DLP) and effective dose for adult and pediatric patients, comparing them with DRL in the literature. The study was performed with data from 293 patients submitted to computed tomography of the skull and 146 of the thorax, divided into age groups 1 어 $1 ; 1-5$; 5- 10; 10- 15; >15). For head, DLP third percentiles and effective doses are mostly higher than DRL in literature, mainly for children from 1 어 15 years $(800$ mGy.cm; 3,3 mSv) and 5\} 10 years (969 mGy.cm; 2,6 $m S v)$. For thorax examination, although DLP are close to the DRL in literature, high variability is revealed, and the effective doses are higher for all ages. In conclusion, the survey of dose indicators in computed tomography examinations and their comparison with DRL constitute an important tool for the optimization of doses in patients.
\end{abstract}

Keywords: dosimetry; computed tomography; effective dose; diagnostic reference level.

\section{Introdução}

A tomografia computadorizada (TC) é uma poderosa ferramenta clínica para o diagnóstico de pacientes devido à capacidade de fornecer dados tridimensionais de alta qualidade resultando em benefícios significativos para a gestão médica, permitindo um diagnóstico mais rápido e preciso, evitando técnicas cirúrgicas intervencionistas ${ }^{1}$. Entretanto, o paciente é sujeito a uma complexa distribuição de dose tridimensionalmente ${ }^{2}$.

Para quantificar o risco da dose em pacientes de exames de TC, é preciso quantificar a medida que é relatada para o risco do paciente. No entanto, em radiologia, a quantidade de dose exposta e absorvida são "unidades de concentração" e não relatam o total da quantidade de radiação absorvida pelo paciente ${ }^{3}$. O DLP (Dose Length Product) fornece uma estimativa razoável de dose de radiação, mas não fornece uma informação sobre o risco da radiação. Para tal efeito, o conceito da dose efetiva foi introduzido pela Comissão Internacional de Proteção Radiológica ou ICRP (International Comission Radiation Protection), para fornecer uma dose baseada no risco da irradiação não homogênea aos pacientes ${ }^{4}$.

Para assegurar que a dose na qual o paciente está sendo submetido seja a mais baixa possível, mas mantenha-se um diagnóstico confiável, determinam-se valores de referência, denominados Níveis de Referência Diagnóstica (NRD), que possibilitam a identificação de doses de radiação que estão elevadas ou fora dos valores estabelecidos como ideais, baseados no terceiro quartil de uma distribuição de doses médias para 
procedimentos específicos, e que são passíveis de investigação para justificação, limitação e otimização. O descritor de dose DLP é o produto

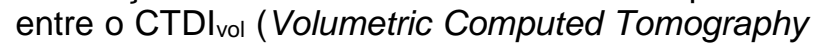
Dose Index) e o comprimento da região irradiada. Estes valores são apenas uma aproximação da dose do paciente ${ }^{5}$ e dependem apenas dos parâmetros selecionados ${ }^{6}$.

A dose efetiva é um descritor de radiação que pode ser utilizado para caracterizar a exposição à radiação para pacientes submetidos a exames de TC, onde os níveis de radiação estão bem abaixo da dose limiar necessária para induzir efeitos determinísticos.

A magnitude da dose efetiva está relacionada com os riscos estocásticos da radiação, como a indução de câncer e a produção de efeitos genéticos ${ }^{7}$.

O objetivo deste estudo é apresentar um levantamento retrospectivo de indicadores de dose em exames de tomografia computadorizada de crânio e tórax, em termos de DLP e dose efetiva, para pacientes adultos e pediátricos, comparandoos com outros NRDs disponibilizados na literatura.

\section{Materiais e Métodos}

Os levantamentos de dados foram realizados no Hospital São Lucas da PUCRS, em dois tomógrafos, Philips Ingenuity Core CT 64 (Philips Medical Systems, Best, the Netherlands) e Siemens Emotion 16 (Siemens Medical Solutions, Erlangen, Germany). Estes dados fazem parte de projeto "Desenvolvimento de um sistema de informação para controle da qualidade e dosimetria de pacientes submetidos a exames de tomografia computadorizada", aprovado pelo Comitê de Ética em Pesquisa (CAAE 18924113000005336, parecer 381.090).

Os dados coletados referem-se aos protocolos de exames de TC mais utilizados para as regiões de crânio e tórax, que foram realizados entre os anos de 2013 a 2015, sem contraste. Os pacientes foram divididos nas seguintes faixas etárias: 0 - 1 ano, 1 † 5 anos, $5 \nmid 10$ anos, $10 \nmid 15$ e $>15$ anos, e foram coletados os seguintes parâmetros: idade, protocolo utilizado, $\mathrm{kV}, \mathrm{mAs}$, CTDlvol e DLP.

Para o protocolo de crânio tem-se as seguintes quantidades de pacientes: 0 - 1 ano: 82 pacientes; 1 - 5 anos: 70 pacientes; $5-10$ anos: 65 pacientes; 10 - 15: 38 pacientes; e >15 anos: 38 pacientes. Para os protocolos de tórax, o número de pacientes é: 0 - 1 ano: 28 pacientes, 1 f 5 anos 41 pacientes, 5 - 10 anos: 11 pacientes, 10 - 15: 20 pacientes e $>15$ anos: 46 pacientes.

A estimativa da dose efetiva foi baseada no estudo de Deak e et al. ${ }^{8}$, que determinou fatores de correções do DLP para o cálculo da dose efetiva em função da tensão, região e a idade dos pacientes, seguindo a recomendação ICRP 103. Os autores desenvolveram fatores de correção para as regiões anatômicas de crânio e tórax, para as tensões de 80 $\mathrm{kV}$ à $140 \mathrm{kV}$.
Com os dados obtidos foi determinado, a partir do terceiro quartil da distribuição de DLP e doses efetivas, valores de comparação para cada um dos tipos de procedimentos e faixas etárias em relação aos NRDs publicados por outros autores.

\section{Resultados}

\subsection{Produto Dose-Comprimento (DLP)}

O gráfico da Figura 1 apresenta os valores de DLP, com a indicação do mínimo e máximo ( $\bullet$ ), primeiro e terceiro quartil (caixa) e mediana $(\diamond)$, para as faixas etárias e regiões anatômicas de crânio.

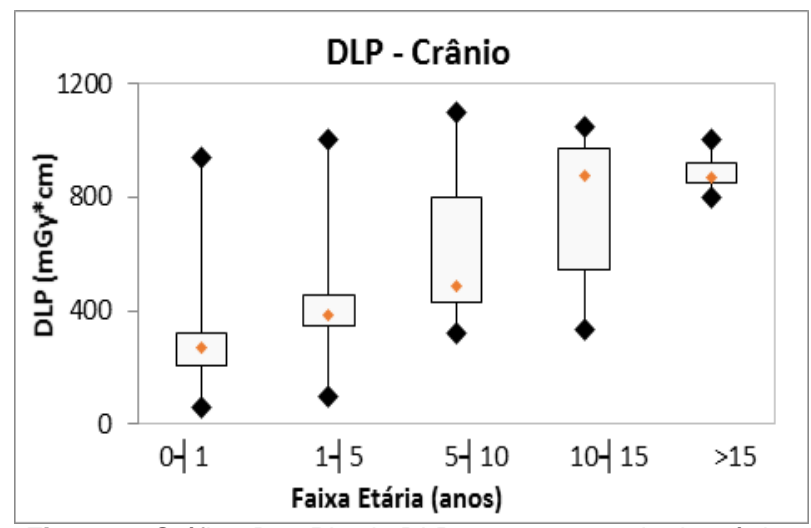

Figura 1: Gráfico Box-Plot do DLP para o protocolo de crânio por faixa etária.

O gráfico da Figura 2 apresenta os valores de DLP, com a indicação do mínimo e máximo ( $\bullet$ ), primeiro e terceiro quartil (caixa) e mediana $(\diamond)$, para as faixas etárias e regiões anatômicas de tórax.

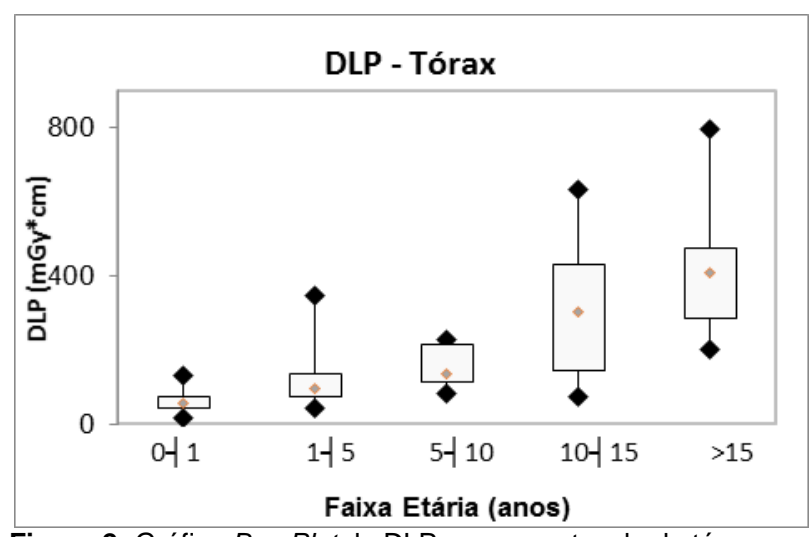

Figura 2: Gráfico Box-Plot do DLP para o protocolo de tórax por faixa etária.

A tabela 1 apresenta os valores de DLP para os protocolos de crânio encontrados na literatura, em comparação com o valor encontrado para o terceiro quartil neste estudo. 
Tabela 1. Comparação entre os valores de DLP obtidos no presente estudo em exames de crânio e os NRDs publicados. Crânio - DLP (mGy*cm)

\begin{tabular}{|c|c|c|c|c|c|}
\hline $\begin{array}{l}\text { Faixa Etária } \\
\text { (anos) }\end{array}$ & 어 1 & $1 \dashv 5$ & $5\} 10$ & 10거 15 & $>15$ \\
\hline Este estudo & 318 & 457 & 800 & 969 & 919 \\
\hline Irlanda $(2012)^{1}$ & 333 & 491 & 608 & 719 & 964 \\
\hline Suíça $(2004)^{2}$ & 270 & 420 & 560 & 1000 & - \\
\hline $\begin{array}{l}\text { Reino Unido } \\
(2005)^{3}\end{array}$ & 270 & 470 & 620 & 930 & - \\
\hline $\begin{array}{l}\text { Alemanha } \\
(2005)^{4}\end{array}$ & 390 & 520 & 710 & 920 & 1100 \\
\hline $\begin{array}{l}\text { Bélgica } \\
(2010)^{5}\end{array}$ & 280 & 473 & 637 & 650 & - \\
\hline $\begin{array}{l}\text { Coréia do Sul } \\
(2015)^{6}\end{array}$ & 545 & 508 & 792 & 947 & - \\
\hline Japão ${ }^{7}(2014)$ & 404 & 624 & 734 & 869 & - \\
\hline
\end{tabular}

A tabela 2 apresenta os valores de DLP para os protocolos de tórax encontrados na literatura, em comparação com o valor encontrado para o terceiro quartil neste estudo.

Tabela 2. Comparação entre os valores de DLP obtidos no presente estudo em exames de tórax e os NRDs publicados anteriormente.

\begin{tabular}{|c|c|c|c|c|c|}
\hline \multicolumn{6}{|c|}{ Tórax - DLP (mGycm) } \\
\hline $\begin{array}{l}\text { Faixa Etária } \\
\text { (anos) }\end{array}$ & 어 1 & $1 \dashv 5$ & $5 \nmid 10$ & 10- 15 & $>15$ \\
\hline Este estudo & 74 & 134 & 136 & 431 & 473 \\
\hline Irlanda (2012) & 73 & 106 & 153 & 237 & 418 \\
\hline Suíça (2004) & 110 & 200 & 220 & 460 & - \\
\hline $\begin{array}{l}\text { Reino Unido } \\
\text { (2005) }\end{array}$ & 200 & 230 & 370 & 580 & - \\
\hline Alemanha (2005) & 28 & 55 & 105 & 205 & 245 \\
\hline Bélgica (2010) & 76 & 111 & 144 & 260 & \\
\hline $\begin{array}{l}\text { Coréia do Sul } \\
(2015)\end{array}$ & 121 & 160 & 226 & 474 & - \\
\hline Japão (2014) & 104 & 127 & 137 & 359 & - \\
\hline
\end{tabular}

\subsection{Dose Efetiva}

O gráfico da Figura 3 apresenta os valores de dose efetiva, com a indicação do mínimo e máximo $(\diamond)$, primeiro e terceiro quartil (caixa) e mediana $(\diamond)$, para as faixas etárias e regiões anatômicas de crânio.

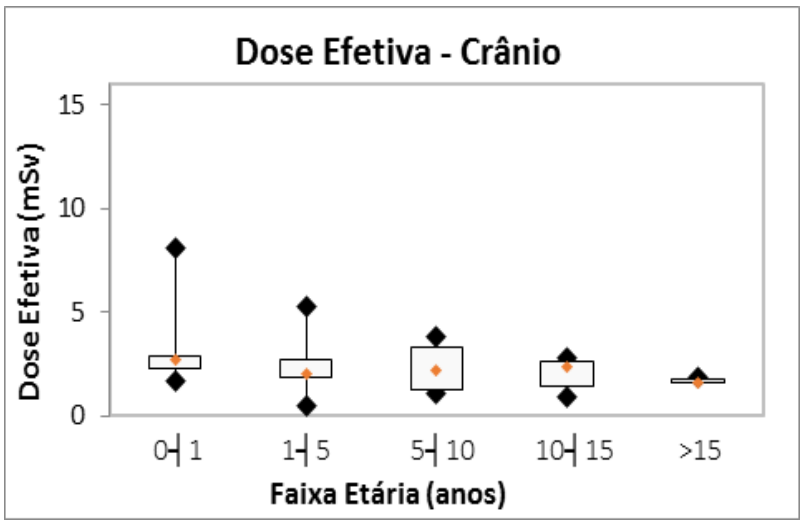

Figura 3: Gráfico Box-Plot da dose efetiva para o protocolo de crânio por faixas etárias.
O gráfico da Figura 4 apresenta os valores de dose efetiva, com a indicação do mínimo e máximo $(\downarrow)$, primeiro e terceiro quartil (caixa) e mediana $(\diamond)$, para as faixas etárias e regiões anatômicas de tórax.

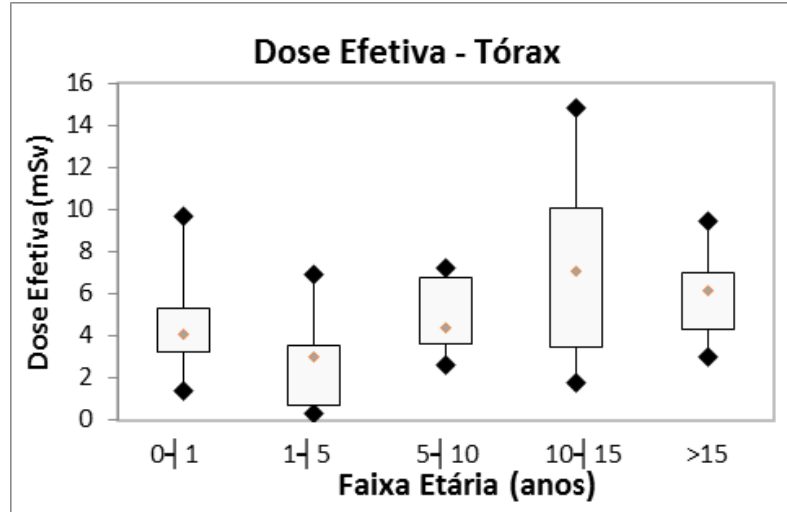

Figura 4: Gráfico Box-Plot da dose efetiva para o protocolo de tórax por faixas etárias.

A tabela 3 apresenta os valores de dose efetiva para os protocolos de crânio encontrados na literatura, em comparação com o valor encontrado para o terceiro quartil neste estudo.

Tabela 3. Comparação entre os valores de dose efetiva obtidos no presente estudo em exames de crânio e os NRDs publicados anteriormente.

\begin{tabular}{|c|c|c|c|c|c|}
\hline \multicolumn{6}{|c|}{ Crânio - Dose Efetiva (mSv) } \\
\hline $\begin{array}{l}\text { Faixa Etária } \\
\text { (anos) }\end{array}$ & 어 1 & $1\} 5$ & $5-10$ & 10- 15 & $>15$ \\
\hline Este estudo & 2,9 & 2,8 & 3,3 & 2,6 & 1,4 \\
\hline $\begin{array}{l}\text { Reino Unido } \\
(2005)\end{array}$ & - & 2,5 & 1,5 & 1,6 & 1,5 \\
\hline Alemanha (2005) & 2,6 & 1,5 & 1,3 & 1,1 & - \\
\hline $\begin{array}{l}\text { Coréia do Sul } \\
\text { (2015) }\end{array}$ & 3,3 & 1,8 & 2,1 & 1,8 & - \\
\hline Japão (2014) & 4,4 & 4,2 & 2,9 & 2,8 & - \\
\hline
\end{tabular}

A tabela 4 apresenta os valores de dose efetiva para os protocolos de tórax encontrados na literatura, em comparação com o valor encontrado para o terceiro quartil neste estudo.

Tabela 4. Comparação entre os valores de dose efetiva obtidos no presente estudo em exames de tórax e os NRDs publicados anteriormente.

\begin{tabular}{lccccc}
\hline \multicolumn{6}{c}{ Tórax - Dose Efetiva (mSv) } \\
\hline $\begin{array}{l}\text { Faixa Etária } \\
\text { (anos) }\end{array}$ & 어1 & $1 \nmid 5$ & $5 \dashv 10$ & $10-15$ & $>15$ \\
\hline $\begin{array}{l}\text { Este estudo } \\
\text { Reino Unido } \\
(2005)\end{array}$ & $\mathbf{5 , 3}$ & $\mathbf{3 , 5}$ & $\mathbf{6 , 8}$ & $\mathbf{1 0 , 1}$ & $\mathbf{7 , 0}$ \\
$\begin{array}{l}\text { Alemanha } \\
(2005)\end{array}$ & 2,7 & 2,0 & 1,5 & 1,1 & - \\
$\begin{array}{l}\text { Coréia do Sul } \\
(2015)\end{array}$ & 2,8 & 2,6 & 2,6 & 3,1 & - \\
Japão (2014) & 4,1 & 3,3 & 2,5 & 4,7 & - \\
\hline
\end{tabular}




\section{Discussão}

\subsection{Produto Dose-Comprimento (DLP)}

\section{Crânio}

Em comparação com os valores da literatura, nosso estudo teve um valor de DLP intermediário $\left(318 \mathrm{mGy}^{*} \mathrm{~cm}\right)$ para a faixa de idade entre 어 1 ano na região anatômica do crânio, estando entre o menor valor $\left(270 \mathrm{mGy}^{*} \mathrm{~cm}\right)$ e o maior valor $(545$ $\mathrm{mGy}^{*} \mathrm{~cm}$ ). Para a faixa etátia entre 1f 5 anos, o valor do DLP (457 mGy* $\left.{ }^{*} \mathrm{~cm}\right)$ foi próximo do menor valor $\left(420 \mathrm{mGy}^{*} \mathrm{~cm}\right)$, sendo o maior valor (624 $\left.\mathrm{mGy}^{*} \mathrm{~cm}\right)$. Entre 5 - 10 anos o valor do DLP (800 $m G y^{*} \mathrm{~cm}$ ) foi maior do que todas as referências, sendo o menor valor de $560 \mathrm{mGy}^{*} \mathrm{~cm}$. Para crianças entre 10 - 15 anos, o valor do DLP foi próximo ao máximo (969 mGy* ${ }^{*} \mathrm{~cm}$ ), de $1000 \mathrm{mGy}^{*} \mathrm{~cm}$, sendo o menor valor de $650 \mathrm{mGy}^{*} \mathrm{~cm}$. Para adultos, considerados acima de > 15 anos, nosso estudo teve um valor de DLP menor do que todas as outras referências $\left(919 \mathrm{mGy}^{*} \mathrm{~cm}\right)$.

Foi possível observar uma grande amplitude interquartil dos valores encontrados para o DLP do protocolo de crânio nas faixas etárias 어 1 ano $5\} 10,10-15$ anos. Apenas o protocolo adulto (> 15 anos não apresenta grande variabilidade.

\section{Tórax}

O nosso estudo teve um valor de DLP intermediário $\left(74 \mathrm{mGy}^{*} \mathrm{~cm}\right)$ para a faixa de idade entre 0 어 1 ano na região anatômica de tórax, sendo o menor valor de $28 \mathrm{mGy}^{*} \mathrm{~cm}$ e o maior valor de 200 $m G y^{*} \mathrm{~cm}$. Para a faixa etária de 1 - 5 anos, o valor do DLP foi intermediário (134 $\left.\mathrm{mGy}^{*} \mathrm{~cm}\right)$, sendo o menor valor de $55 \mathrm{mGy}^{*} \mathrm{~cm}$ e o maior valor 220 $m G y^{*} \mathrm{~cm}$. Entre $5-10$ anos, o valor do DLP (136 $\left.\mathrm{mGy}^{*} \mathrm{~cm}\right)$ foi próximo ao menor valor $\left(105 \mathrm{mGy}^{*} \mathrm{~cm}\right)$, sendo o maior valor de $370 \mathrm{mGy}^{*} \mathrm{~cm}$. Para a faixa de idade entre $10 \dashv 15$ anos, o valor do DLP foi intermediário (431 $\left.\mathrm{mGy}^{*} \mathrm{~cm}\right)$, sendo o menor valor de $205 \mathrm{mGy}^{*} \mathrm{~cm}$ e o maior valor $580 \mathrm{mGy}^{*} \mathrm{~cm}$. Para adultos (> 15 anos), o valor do DLP foi maior do que todas as referências $\left(478 \mathrm{mGy}^{*} \mathrm{~cm}\right)$, sendo o menor valor de $245 \mathrm{mGy}^{*} \mathrm{~cm}$.

Para o protocolo de tórax, observa-se que uma grande amplitude interquartil dos valores de DLP ocorre para a faixa etária 10-15 anos. Possivelmente essa variação deve-se às grandes diferenças estruturais da região torácica nessa faixa etária.

\subsection{Dose Efetiva \\ Crânio}

O presente estudo apresentou um valor de dose efetiva intermediário $(2,9 \mathrm{mSv})$ para a faixa de idade entre 어 1 ano na região anatômica do crânio, sendo o menor valor da literatura de 2,6 mSv e o maior de $4,4 \mathrm{mSv}$. Para idades entre 1 f 5 anos, a dose efetiva foi intermediária $(2,8 \mathrm{mSv})$ sendo o menor valor de 1,5 mSv e o maior valor de 4,2 mSv. Para a faixa de idade entre 5 - 10 anos, nosso estudo apresentou a dose efetiva mais elevada (3,3 mSv), quando comparado com as outras referências. Para crianças entre 10 - 15 anos, o valor de dose efetiva foi intermediário (2,6 mSv), sendo o menor valor 1,1 $\mathrm{mSv}$ e o maior valor $2,8 \mathrm{mSv}$. Para a faixa de idade acima de $>15$ anos, nosso estudo teve a menor dose efetiva $(1,4 \mathrm{mSv})$ em relação às referências.

A mesma variabilidade interquartil dos valores encontrados para o DLP do protocolo de crânio é observada para a dose efetiva dessa região anatômica, particularmente para as crianças.

\section{Tórax}

A dose efetiva média no tórax determinada em nosso estudo foi a maior (5,3 mSv) encontrada na literatura para a faixa de idade entre 어 1 ano, chegando a praticamente o dobro do menor valor $(2,7 \mathrm{mSv})$. Para a faixa entre 1 f 5 anos, a dose efetiva foi intermediária (3,5 mSv), sendo o menor valor de $1,0 \mathrm{mSv}$ da e o maior 6,3 mSv. As faixas de idades entre $5 \dashv 10$ e entre $10 \nmid 15$ anos também apresentaram as maiores doses efetivas - $6,8 \mathrm{mSv}$ e 10,1 mSv, respectivamente - considerando as referências comparadas. Para a população adulta, acima de > 15 anos, nosso estudo também apresentou uma dose efetiva maior $(7,0 \mathrm{mSv})$ que a outras referências, sendo o maior valor foi do Reino Unido $(7,0$ vs 5,8$) \mathrm{mSv}$. Observa-se uma grande amplitude interquartil dos valores de dose efetiva para a faixa etária $10-15$ anos, como identificado anteriormente para o DLP da mesma região anatômica.

Deve-se destacar que a estimativa de DLP e da dose efetiva para os pacientes pediátricos neste estudo, tanto para a região do crânio como do tórax, pode estar sendo subestimada pela falta de phantoms que representem adequadamente pacientes pediátricos e recém-nascidos. De acordo com Brink e Morin ${ }^{9}$, o uso de phantom de $32 \mathrm{~cm}$ para pacientes pediátricos resulta numa subestimação da dose recebida, pois os phantoms de PMMA são desenvolvidos para o imageamento de pacientes adultos (diâmetro de $16 \mathrm{~cm}$ para crânio e $32 \mathrm{~cm}$ para abdômen e tórax). Infelizmente, estes dois tamanhos não representam nem as pequenas dimensões de recém-nascidos, nem as dimensões de pacientes adultos obesos.

Alguns fabricantes vêm utilizando o phantom de $16 \mathrm{~cm}$ para abdômen e tórax em pacientes pediátricos, mas ainda não há um consenso sobre o uso de phantoms de crânio pediátrico. O estudo de Nickoloff, Dutta e $\mathrm{Lu}^{8}$, ao avaliar o uso de phantoms de diâmetros de $6 \mathrm{~cm}, 10 \mathrm{~cm}, 16 \mathrm{~cm}, 24$ $\mathrm{cm}$ e $32 \mathrm{~cm}$, mostrou que a dose no phantom de 6 $\mathrm{cm}$ pode ser de 3 a 6 vezes maior, quando comparado com o de $32 \mathrm{~cm}$.

Assim, os resultados mostram a importância da determinação dos valores de mAs e kV para cada paciente, dependendo da região estudada, idade, e espessura, reforçado por estudos mais atuais ${ }^{10-12}$.

Os protocolos avaliados deveriam ser otimizados, particularmente para pacientes pediátricos, pois os mesmos possuem um maior risco de exposição à radiação ionizante ${ }^{8}$. 


\section{Conclusões}

Conclui-se que o levantamento de indicadores de dose em exames de tomografia computadorizada e sua comparação com níveis de referência de diagnóstico constituem uma importante ferramenta para a otimização de doses em pacientes, endossado por diversas organizações profissionais e reguladoras, incluindo a ICRP, Colégio Americano de Radiologia (ACR), Associação Americana de Físicos em Medicina (AAPM), Reino Unido (UK) Proteção da Saúde Agência, Agência Internacional de Energia Atômica (AIEA) e Comissão Europeia (CE).

Estes levantamentos são particularmente relevantes para exames realizados em pacientes pediátricos, em função da maior radiossensibilidade nessa faixa etária.

\section{Agradecimentos}

À Fundação de Apoio à Pesquisa do Estado do Rio Grande do Sul (FAPERGS) pelo apoio financeiro.

\section{Referências}

1. Foley, S. J., Mcentee, M. F. \& Rainford, L. A. Establishment of CT diagnostic reference levels in Ireland. British Journal of Radiology. 85, 1390-1397 (2012).

2. Aroua, A. et al. Adult reference levels in diagnostic and interventional radiology for temporary use in Switzerland. Radiation Protection Dosimetry 111, 289-295 (2004).

3. P C Shrimpton, M C Hillier,M A Lewis, M. D. Doses from Computed Tomography (CT) Examinations in the UK - 2003 Review. Natl. Radiol. Prot. Board 57, 1-107 (2005).

4. Galanski, M., Nagel, H. D. \& Stamm, G. Paediatric CT Exposure Practice in the Federal Republic of Germany: Results of a Nation-wide Survey in 2005/06. (2005).

5. Buls, N. et al. CT paediatric doses in Belgium : a multi-centre study. Belgian Fed. Agency Nucl. Control (2010). at <http://www.fanc.fgov.be/GED/00000000/2400/2449.pdf>

6. Hwang, J.-Y. et al. A Survey of Pediatric CT Protocols and Radiation Doses in South Korean Hospitals to Optimize the Radiation Dose for Pediatric CT Scanning. Medicine (Baltimore). 94, 1-9 (2015).

7. Here, S. T. Optimized Pediatric Ct Dose At a Tertiar Y Children ' S Hospital in Japan: a 4-Year Single Center Analysis. 0, 1-12 (2016).

8. Nickoloff, E. L., Dutta, A. K. \& Lu, Z. F. Influence of phantom diameter, $\mathrm{kVp}$ and scan mode upon computed tomography dose index. Medical Physics. 30, 395-402 (2003).

9. Brink, J. a \& Morin, R. L. Size-specific dose estimation for CT: how should it be used and what does it mean? Radiology 265, 666-8 (2012).

10. Suess, C. \& Chen, X. Dose optimization in pediatric CT: Current technology and future innovations. Pediatric Radiology 32, 729-734 (2002).

11. Reid, J., Gamberoni, J., Dong, F. \& Davros, W. Optimization of $\mathrm{kVp}$ and $\mathrm{mAs}$ for pediatric low-dose simulated abdominal CT: Is it best to base parameter selection on object circumference? AJR. 195, 1015-1020 (2010).

12. Al Mahrooqi, K. M. S., Ng, C. K. C. \& Sun, Z. Pediatric Computed Tomography Dose Optimization Strategies: A Literature Review. Journal of Medical Imaging and Radiation Sciences 46, 241-249 (2015).

\section{Contato:}

Giordana Salvi de Souza

Av. Ipiranga 6681, Pr. 10

90619-900 Porto Alegre-RS

E-mail: giordana.souza@acad.pucrs.br 